\title{
Um caso de leishmaniose cutâneo-mucosa tratado com sucesso com baixa dose de antimonial pentavalente
}

\author{
A case of American tegumentar leishmaniasis \\ successfuly treated with low dose of \\ pentavalent antimonial
}

\author{
Valdir Sabbaga Amato, Luciana Silveira de Oliveira, Anita Compos Mendonça \\ Silva, Flávia Ribeiro Machado, Juliane Gomes de Paula Amato, \\ Antônio Corlos Nicodemo e Vicente Amato Neto
}

\begin{abstract}
Resumo Os autores relatam um caso de leishmaniose cutâneo-mucosa em uma paciente de 89 anos, diabética e hipertensa, tratada inicialmente com alopurinol por 10 meses não havendo cicatrização das lesões. Posteriormente, recebeu antimoniato de N-metil glucamina (glucantime) por 4 dias, na dose total de 2.380mg do Sbv, mas desenvolveu cardiotoxicidade e hipocalemia, sendo suspenso o tratamento, entretanto, evoluiu com regressão clínica total das lesões, apesar de ter recebido pequena dose desta medicação.
\end{abstract}

Palavras-chaves: Leishmaniose tegumentar americana. Leishmaniose cutâneo-mucosa. Alopurinol. Antimonial pentavalente.

\begin{abstract}
The authors report a case of a 89 years-old woman with mucocutaneous leishmaniasis and previous diabetes mellitus and high blood pression, who had been treated with allopurinol for 10 months without healing of lesions. Afterwards, she has been treated with meglumine antimonate, "glucantime" for 4 days, with a total dose 2,380mg of Sbv, but developed cardiacs side effects and hypokalemia, hence the treatment was wihtdrawn. However, this patient developed total clinical regression of lesions, in spite of she has been recieved low dose of this drug.
\end{abstract}

Key-words: American tegumental leishmaniasis. Mucocutaneous leishmaniasis. Allopurinol. Pentavalent antimonials.

\footnotetext{
Departamento de Doenças Infecciosas e Parasitárias do Hospital das Clínicas da Faculdade de Medicina da Universidade de São Paulo, São Paulo, SP.

Endereço para correspondência: Dr. Valdir Sabbaga Amato. Deptº de Doenças Infecciosas e Parasitárias/FM/USP. Av. Dr. Enéas de Carvalho Aguiar 255, 05403-900 São Paulo, SP. Fax: (011) 881-8158.

Recebido para publicação em 09/05/97.
} 
As leishmanioses são enfermidades causadas por várias espécies de protozoários digenéticos da ordem Kinetoplastida, família Trypanomastidae, e gênero Leishmania, que acometem o homem e diferentes espécies de animais silvestres e domésticos. Nas Américas a leishmaniose tegumentar americana (LTA) é transmitida entre os animais e o homem pela picada das fêmeas de diversas espécies de flebótomos (Diptera, Psycodidae, Phlebotominae) dos gêneros Lutzomyia e Psychodopygus 6. No Brasil, a doença é um grande problema de saúde pública, devido a sua grande distribuição demográfica e ao grau de morbidade ocasionada pelas suas formas cutânea ou cutâneo-mucosa12. Quando a LTA é ocasionada pela Leishmania (Viannia) braziliensis pode ocorrer acometimento de mucosas, incluindo faringe, laringe e traquéia, podendo ocasionar a morte do paciente por aspiração ou insuficiência respiratória1. Este relato de caso trata-se de uma paciente com leishmaniose cutâneo-mucosa (LCM), que apresentou resposta terapêutica a pequena dose de antimonial pentavalente e antecedente de falha ao tratamento prolongado com alopurinol.

\section{RELATO DO CASO}

Paciente JMS, 89 anos, feminina, branca, natural de Rio do Peixe, no Estado do Espírito Santo e procedente de São Paulo, Capital. Atendida no Ambulatório de LTA da Divisão de Clínica de Moléstias Infecciosas e Parasitárias do Hospital das Clínicas da Faculdade de Medicina da Universidade de São Paulo (HCFMUSP), em março de 1996, com queixa de lesão ulcerada em região nasal e lábio superior há três anos, ocasionando limitação da alimentação e fala. Ao exame físico, foi observada lesão ulcerada em lábio superior, medindo cerca de $5 \mathrm{~cm}$ de diâmetro no seu maior eixo, com fundo granuloso, formato ovalado, bordas enduradas, base infiltrada e presença de abundante secreção purulenta (Figura 1). Ao exame otorrinolaringológico, observou-se perfuração de septo nasal, com as bordas da cartilagem nasal apresentando-se hiperemiadas e aspecto granulomatoso. O

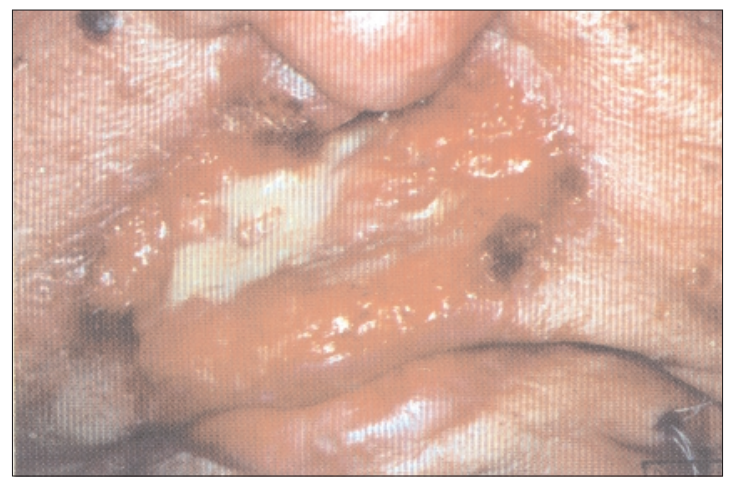

Figura 1 - Aspecto da lesão, anteriormente ao tratamento. diagnóstico de LCM foi realizado através de reação intradérmica de Montenegro (nódulo de $40 \mathrm{~mm}$ ), sorologia pela técnica de imunofluorescência indireta (título de 1:128) e exame anatomopatológico obtido de material da biópsia da lesão de pele e septo nasal (raras formas amastigotas). Por se tratar de paciente com alterações eletrocardiográficas, constituídas por extrassístoles ventriculares, bem como glicemias de jejum compatíveis com diagnóstico de diabetes mellitus, optou-se pela introdução de alopurinol, na posologia de $20 \mathrm{mg} / \mathrm{kg} / \mathrm{dia}$, o qual a doente utilizou por 10 meses, sem qualquer alteração no aspecto clínico ou ao exame otorrinolaringológico da lesão. Em janeiro de 1997, a doente foi internada na Enfermaria da Divisão de Clínica de Moléstias Infecciosas do HC-FMUSP, pois a lesão estava ocasionando desconforto intenso, inclusive com dificuldade para alimentação.

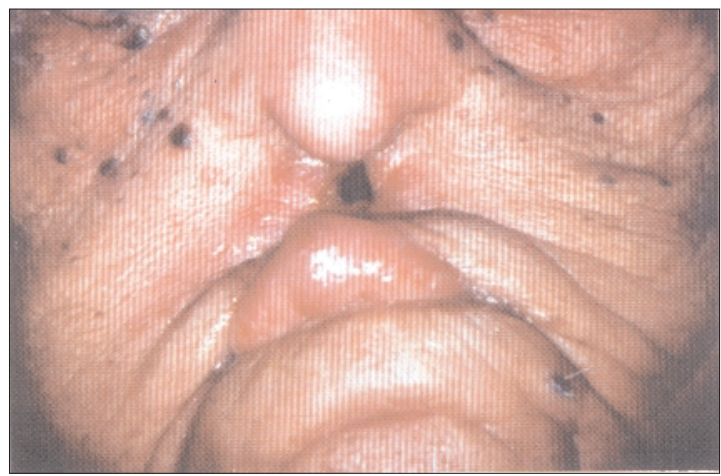

Figura 2 - Aspecto da lesão, posteriormente ao tratamento. 
Apresentava à internação, níveis discretamente elevados de dosagens séricas de uréia e creatinina, 74 e 1,8mg/dl respectivamente, glicemia de jejum de $150 \mathrm{mg} / \mathrm{dl}$ e as alterações eletrocardiográficas descritas anteriormente. As dosagens séricas de sódio e potássio, transaminases e o hemograma completo e plaquetas apresentavam-se dentro dos limites da normalidade. Foi iniciado o antimoniato de $\mathrm{N}$ metil glucamina (glucantime) na posologia de $10 \mathrm{mg} / \mathrm{kg} / \mathrm{dia}$ de $\mathrm{Sbv}$, sendo realizado eletrocardiograma diariamente após infusão da medicação. Após receber dose total de $2380 \mathrm{mg}$ de $S b \vee$ por via endovenosa, em 4 dias, a doente apresentou outras alterações eletrocardiográficas, consistindo de marcapasso atrial mutável, hipocalemia e discreto aumento das transaminases séricas. Devido a estes efeitos adversos, foi suspenso o tratamento, sendo encaminhada para tratamento ambulatorial. Em março de 1997, retorna ao Ambulatório apresentando regressão clínica da lesão cutânea (Figura 2). A avaliação otorrinolaringológica observou destruição da parede inferior da pirâmide nasal, dos cornetos e parede lateral do nariz, com exposição do seio maxilar e ausência de sinais de atividade da lesão. A paciente continua em acompanhamento ambulatorial regularmente.

\section{DISCUSSÃO}

As drogas de primeira escolha no tratamento da LTA são os antimoniais pentavalentes ${ }^{13}$. Estas medicações, e especificamente o antimoniato de N-metil glucamina, habitualmente utilizado em nosso país, apresentam vários efeitos colaterais tais como: artralgia, mialgia, inapetência, náusea, vômito, sensação de plenitude gástrica, epigastralgia, pirose, dor abdominal, prurido, febre, fraqueza, cefaléia, tontura e alterações eletrocardiográficas doses dependentes, tais como inversão de ondas $\mathrm{T}$ e prolongamento do intervalo QT. Porém arritmias fatais são raras; insônia, choque pirogênico, edema, hepatite com aumento de transaminases e fosfatase alcalina, pancreatite, insuficiência renal aguda por alteração da liberação do hormônio antidiurético e toxicidade direta sobre as células tubulares. Devido a estas alterações, os antimoniais não são indicados em cardiopatas, nefropatas e hepatopatas7 91013 19. A posologia do (glucantime) recomendada para o tratamento da LCM, é de $20 \mathrm{mg} / \mathrm{kg} / \mathrm{dia}$ de Sbv por 30 dias $^{4}$. Importante ressaltar, que mesmo com este esquema terapêutica as falhas do tratamento com os antimoniais, principalmente no que se refere a forma mucosa da LTA são freqüentes10 14.

Neste caso relatado, não foi possível introduzir as duas alternativas mais comumente utilizadas para o tratamento da LTA, ou seja, a pentamidina, pois a doente era diabética, e a anfotericina $B$ devido a sua cardiotoxidade e nefrotoxidade, pois que a paciente já possuía alterações previamente a terapêutica13 17.

Estudos com medicações que possuem menor toxicidade utilizadas para a terapêutica da LTA abrangem o cetoconazol, itraconazol e a dapsona. O primeiro parece não possuir atividade na forma mucosa causada pela $L$. V. braziliensis, embora sejam necessários maior número de trabalhos, e quanto aos dois últimos, somente foram usados isoladamente para a forma cutânea. Diante do exposto, não os utilizamos na nossa doente4 5818 .

Devido a evidências que o alopurinol pode inibir o crescimento de leishmânias in vitro e em culturas de células 211 , e de estudos utilizando esta medicação no tratamento da LTA, em formas cutâneas e mucosa desta enfermidade, com algum resultado positivo11 1516 , optou-se por tratar a paciente com a droga em questão pela sua menor toxicidade, mas não houve qualquer sucesso. O tempo de uso do alopurinol por 10 meses, foi justificado por estarmos aguardando algum êxito na terapêutica, diante da dificuldade de introduzir outro medicamento. Não nos parece ter ocorrido qualquer efeito sinérgico entre o alopurinol e o antimônio pentavalente, pois o alopurinol é rapidamente eliminado do plasma, sendo sua meia-vida de 2 a 3 três horas ${ }^{19}$. Além do mais, as duas drogas não foram utilizadas concomitantemente.

Embora haja relato anterior com doentes apresentando a forma cutânea da doença que responderam a doses menores que as habitualmente recomendadas de antimoniais 3 , a nossa paciente possuía lesão concomitante de mucosa, sabidamente de difícil tratamento. Neste caso, ressaltamos dois aspectos, primeiro a dificuldade no tratamento da LCM diante de pacientes cardiopatas e nefropatas, devido à falta de opções terapêuticas, com baixa toxicidade, bem como a falta de drogas com baixa toxicidade, e segundo a falta de resposta de resposta terapêutica ao alopurinol. Enfatizamos também, o diferente comportamento que os doentes podem apresentar diante da LCM; alguns não respondem aos antimoniais, mesmo com doses altas e período de tratamento prolongado e 
outros doentes não cicatrizam a(s) lesão(ões) após o uso de várias medicações. Paradoxalmente, a paciente em questão respondeu a baixa dose de (glucantime), o que remonta à necessidade de uma melhor compreensão sobre as relações parasito-hospedeiro desta enfermidade, bem

\section{REFERÊNCIAS BIBLIOGRÁFICAS}

como a sua própria fisiopatogenia.

1. Amato VS, Boulos MIC, Amato Neto V, Filomeno LTB. Utilização do tubo em "T" de silicone para tratamento de um caso de leishmaniose tegumentar americana com traqueomalácia. Revista da Sociedade Brasileira de Medidicina Tropical 28:129-130, 1995.

2. Berens RL, Mar JJ, Nelson DJ, LaFon SW. Antileishmanial effect of allopurinol and allopurinol ribonucleoside on intracellular forms of Leishmania donovani. Biochemical Pharmacology 29:2397-2398, 1980.

3. Costa JML, Marsden PD. Low dose glucantime therapy in Leishmania Viannia braziliensis (LVb) infections. Revista da Sociedade Brasileira de Medicina Tropical 21:85-86, 1988.

4. Droga J. A double-blind study on the efficacy of oral dapsone in cutaneous leishmaniasis. Transactions of the Royal Society of Tropical Medicine and Hygiene 85:212-213, 1991

5. Droga J, Aneja N, Lal BB, Mishra SN. Cutaneous leishmaniasis in India, Clinical experience with itraconazole. International Journal of Dermatology 29:661-662, 1990.

6. Furtado TA. Leishmaniose Americana. In: Amato Neto V, Baldy JLS (eds) Doenças Transmissíveis, $3^{\text {a }}$ edição, Sarvier, São Paulo p.553-557, 1989.

7. Gasser Jr RA, Magill AJ, Oster CN, Franke ED, Grögl $M$, Berman JD. Pancreatitis induced by pentavalent antimonial agents during treatment of leishmaniasis. Clinical of Infectious Diseases 18:83-90, 1994.

8. Jolliffe DS. Cutaneous leishmaniasis from Belize treatment with ketoconazole. Clinical Experimental. Dermatology 11:62-68, 1986.

9. Marsden PD. Pentavalent antimonials: old drugs for new diseases. Revista da Sociedade Brasileira de Medicina Tropical 18:187-195, 1985.

10. Marsden PD. Mucosal leishmaniasis ("espundia" Escomel, 1911). Transactions Royal Society of
Tropical Medicine and Hygiene 80:859-876, 1986.

11. Martinez S, Marr JJ. Allopurinol in the treatment of American cutaneous leishmaniasis. New England Journal of Medicine 326:741-744, 1992.

12. Marzochi MCA. Leishmanioses no Brasil. Journal Brasileiro de Medicina 63:82-104, 1992.

13. Ministério da Saúde, Secretaria Nacional de Programas Especiais de Saúde, Divisão Nacional de Dermatologia Sanitária. (Portaria n:02). Diário Oficial, Brasília, DF, 3 de janeiro de 1990.

14. Oliveira MRF, Macêdo VO, Carvalho M, Barral A, Marotti JG, Bittencourt A, Abreu MVA, Orge MGO, Lessa HA, Marsden PD. Estudo evolutivo da leishmaniose mucosa (7 a 17 anos se seguimento) causada por Leishmania (Viannia) braziliensis em Três Braços, Bahia. Revista da Sociedade Brasileira de Medicina Tropical 28:325-32, 1995.

15. Saenz RE, Paz HM, Johnson CM, Marr JJ, Nelson DJ, Pattishall KH, Rogers MD. Treatment of american leishmaniasis with orally administered allopurinol riboside. The Journal of Infectious Diseases 160:153158, 1989.

16. Sampaio RN, Marchán EM, Venexat A, Tristão RJ, Marsden PD. Combined antimonial alluporinol therapy in mucosal leishmaniasis. Memórias do Instituto Oswaldo Cruz 85:373-374, 1990.

17. Sands M, Kron MA, Brown RB. Pentamidine: a review. Reviews of Infectious Diseases 7:625-634, 1985.

18. Urcuyo FG, Zaias N. Oral ketoconazole in the treatment of leishmaniasis. International Journal of Dermatolology 21:414-416, 1982.

19. Webster LT. Drogas usadas na quimioterapia por protozoários. In: Gilman AG, Goodman LS, Gilman A (eds) As Bases Farmacológicas da Terapêutica, 7a edição, Guanabara Koogan, Rio de Janeiro p.695700, 1983. 